\title{
Fermilab
}

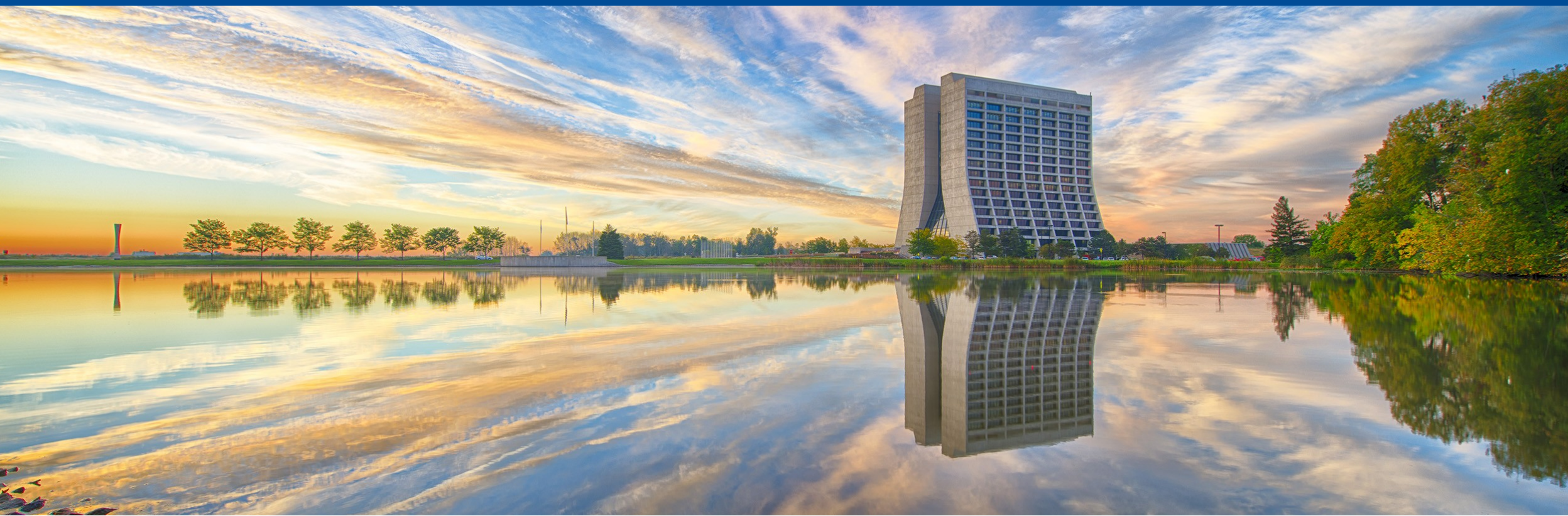

\section{Exploring the Great Pyramid}

Tabitha Welch

2020 SIST Intern; University of Chicago

Supervisor: Dr. Alan Bross

3 August 2020
This manuscript has been authored by Fermi Research Alliance, LLC under Contract No. DE-AC02-07CH11359 with the U.S. Department of Energy, Office of Science, Office of High Energy Physics.

THE UNIVERSITY OF

UINIVERITY


Outline:

I. Historical Background

II. Motivation

III. EGP Detector Proposal

IV. Independent Monte Carlo Study

- Using dE/dx to Determine Incident Muon Momentum

- Muon Position/Angular Resolution 


\section{Historical Background: Cosmic-Ray Muon Radiography}

\section{Search for Hidden Chambers} in the Pyramids

The structure of the Second Pyramid of Giza is determined by cosmic-ray absorption.

Luis W. Alvarez, Jared A. Anderson, F. El Bedwei, James Burkhard, Ahmed Fakhry, Adib Girgis, Amr Goneid, Fikhry Hassan, Dennis Iverson, Gerald Lynch, Zenab Miligy, Ali Hilmy Moussa, Mohammed-Sharkawi, Lauren Yazolino

The three pyramids of Giza are situated a few miles southwest of Cairo, Egypt. The two largest pyramids stand
within a few hundred meters of each within a few hundred meters of each other. They were originally of almost exactly the same height (145 meters), but the Great Pyramid of Cheops has a slightly larger square base (230 meters
on a side) than the Second Pyramid of on a side) than the Second Pyramid of phophren (215.5 meters on a side). A photograph of the pyramids at Giza
is shown as Fig. 1. Figure 2 shows the is shown as Fig. 1. Figure 2 shows the
elevation cross sections of the two elevation cross sections of the two
pyramids and indicates the contrast in pyramids and indicates the contrast in
architectural design. The simplicity of architectural design. The simplicity of Chephren's pyramid, compared with
the elaborate structure of his father's Great Pyramid, is explained by archeologists in terms of a "period of ex-
perimentation," ending with the conperimentation," ending with the con-
struction of Cheops's pyramid (I). (The mun in the 9th century A.D., almost 3400 years after its construction. Of our group only Ahmed Fakhry (author
of The Pyramids, professor emeritus of of The Pyramids, professor emeritus of archeology, University of Cairo, and member of the Supreme Council of
Archeology, Cairo) was trained in arArcheology, Cairo) was trained in archeology. As laymen, we thought it not unlikely that unknown chambers might still be present in the limestone above the "Belzoni Chamber," which is near the center of the base of Chephren's bers had survived undetected for 4500 years. [We learned later that such ideas had occurred to early 19 th-century investigators (2), who blasted holes in the
pyramids with gunpowder in attempts pyramids with gunpowder
to locate new chambers.]

In 1965 a proposal to probe the
locate new chamber.] In 1965 a proposal to probe the
Second Pyramid with cosmic rays (3) of the thickness of rock overlying an underground powerhouse in Australia's Snowy Mountains Scheme (5)].

The favorable response to the proposal led to the establishment by the United Arab Republic and the United States of America of the Joint U.A.R. U.S.A. Pyramid Project on 14 June 1966. Cosmic-ray detectors were installed in the Belzoni Chamber of the Second Pyramid at Giza in the spring of 1967 by physicists from the Ei Shams University and the University of California, in cooperation with archeologists from the U.A.R. Deparment of Antiquities. Initial operatio had been scheduled for the middle of June 1967, but for reasons beyond ou control the schedule was delayed for several months. In early 1968 cosmic ray data began to be recorded on mag netic tape in our laboratory building a few hundred meters from the two argest pyramids. Since that time we have accumulated accurate angula measurements on more than a millio cosmic-ray muons that have penetrate an average or about 100 meters of limestone on their way to the detectors in the Belzoni Chamber.

Prof of the Method

Before any new technique is used in an exploratory mode, it is essentia that the capabilities of the technique be demonstrated on a known system. We gave serious consideration to a proposal that the cosmic-ray detectors be tested first in the Queen's Chamber of the Great Pyramid, to demonstrate Gallery could be detected. But this
- Feb. 1970: Alvarez et al. publish study of Khafre's Pyramid using cosmic rays

- Nov. 2017: ScanPyramids Collaboration publishes Nature article announcing discovery of new void above Grand Gallery in the Great Pyramid of Khufu 


\section{Motivation: Why do it again?}

- Alvarez \& ScanPyramids teams both focused on finding new voids

- ScanPyramids succeeded

- Many other open questions!

- Why did subsequent pyramids lack the complex internal structure that characterizes Khufu's tomb?

- What can be learned about the details of this structure from high-resolution tomographic imaging?

- Distinguish not only voids from solid stone, but also subtler variations in density

- Gain insight regarding construction techniques - how was the Great Pyramid built?

- Reveal unknown unknowns? 


\section{Muon Tomography}

- Unlike previous projects, EGP will apply true tomographic imaging \& reconstruction

- Cosmic particles like protons interact w/ upper atmosphere $\rightarrow$ muons

- When they reach Earth's surface, many muons still have enough energy to penetrate massive structures - like the Great Pyramid

- Rate of muons that make it to the other side, measured as a function of angle, yields information about the structure

- Repeating such measurements at many different locations around pyramid permits full 3D imaging of its interior!

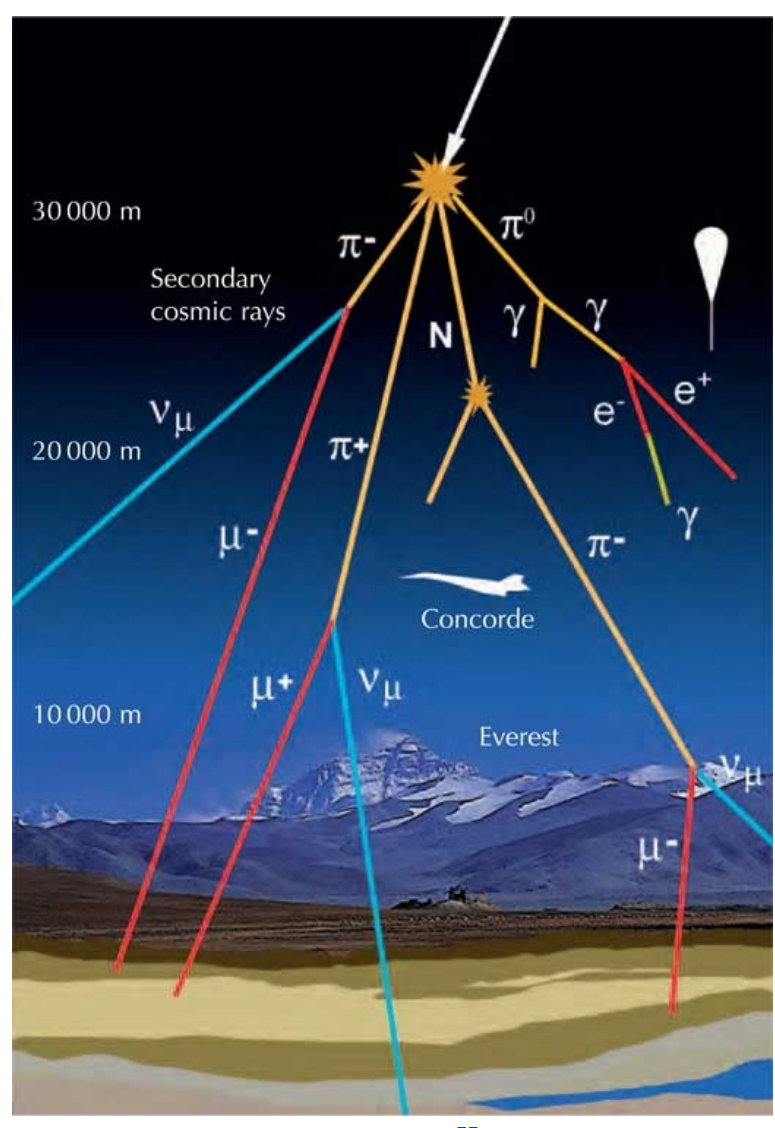




\section{EGP Detector Proposal}

The Great Pyramid of Khufu by the numbers:

- Total Mass: 5,800,000 tons

- Number of Blocks: 2,300,000

- Angle of Slope: $51^{\circ} 50^{\prime} 40^{\prime \prime}$

- Queen Pyramids: 3

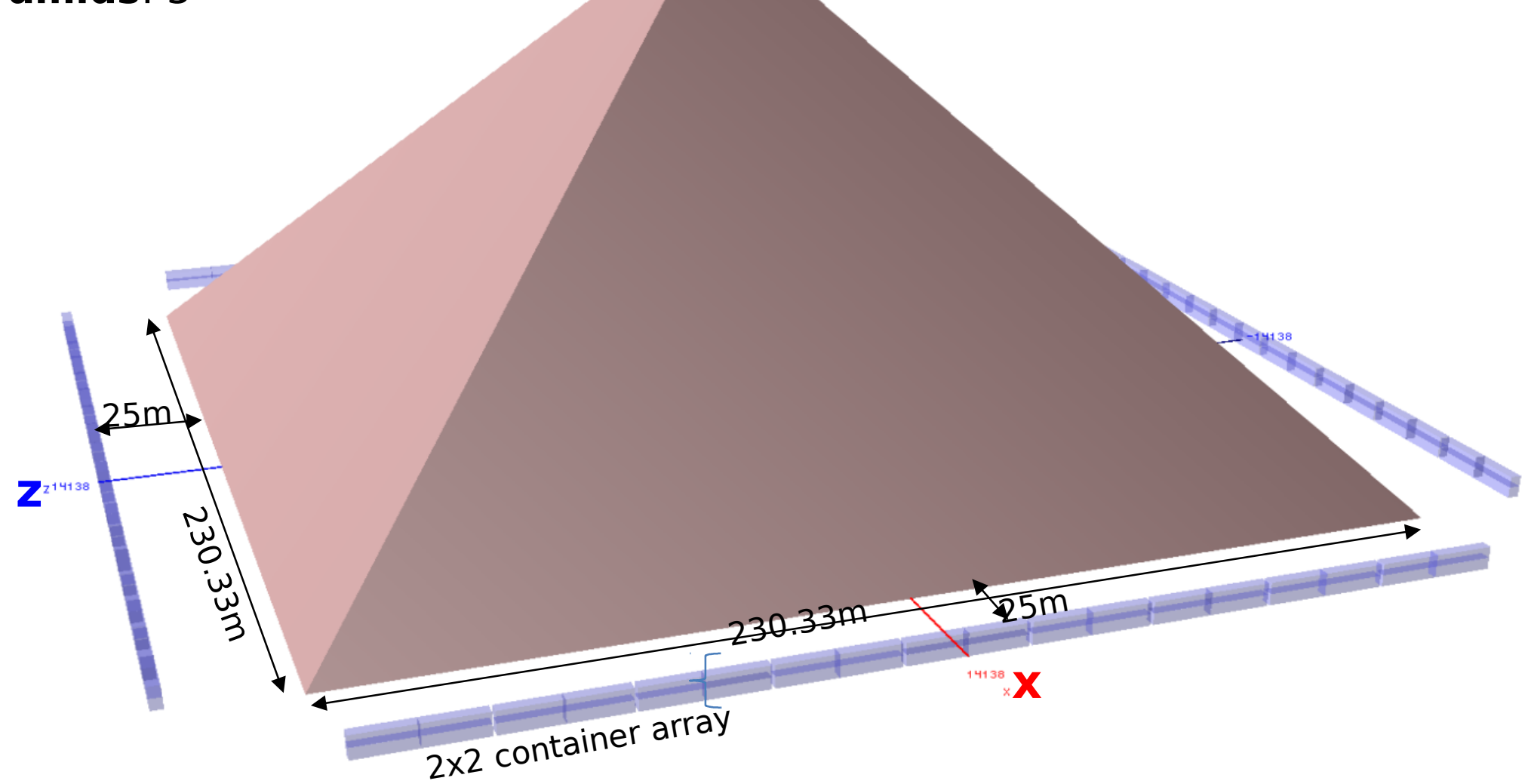




\section{EGP Detector Proposal}

- Detectors housed in 40-ft. steel shipping containers

- Field 8 such containers in $2 \times 2$ arrays

- Move around pyramid perimeter for tomographic image reconstruction

- On each container wall, 2 arrays of extruded scintillator strips

- In each array, 6 vertical modules (40 strips each) \& 3 horizontal modules (40 strips each) for a total of $\sim 1400$ strips

- Vertical strips $2.4 \mathrm{~m}$. in length, horizontal strips $\sim 4.8 \mathrm{~m}$.; walls separated by $2 \mathrm{~m}$.

- Scintillator strips:

- Emit light when exposed to ionizing radiation (here, muons)

- Embedded w/ wavelengthshifting fibers to collect a fraction of this light \& transmit it to SiPMs

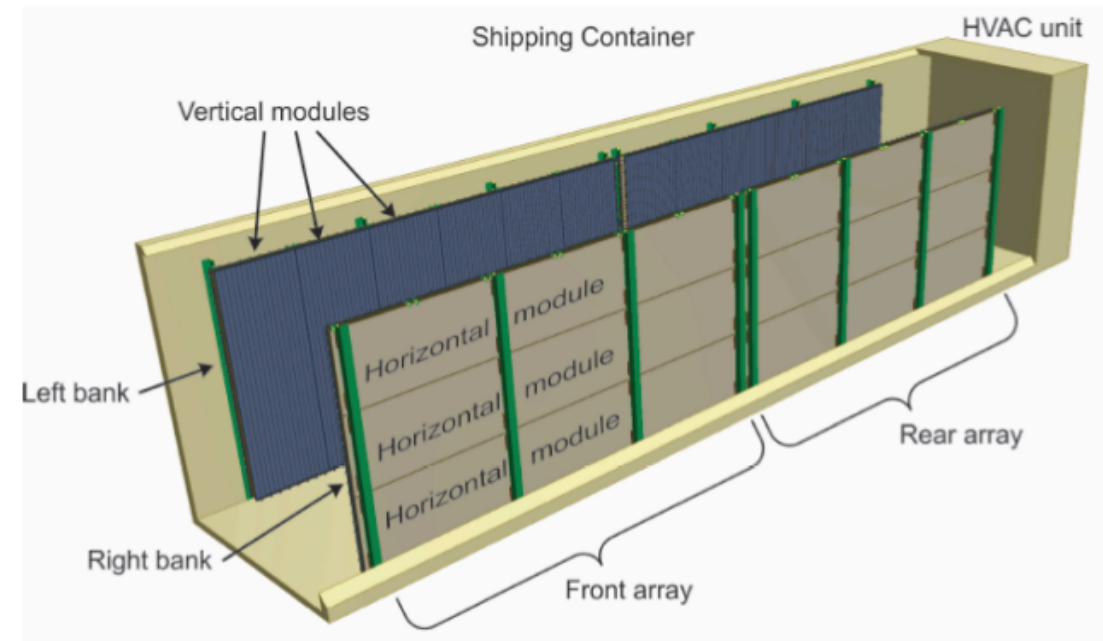




\section{Independent Monte Carlo Study}

- Goal: isolate problem of detector design in a stand-alone GEANT4 simulation

- Two primary questions:

- What is the necessary detector resolution?

- Limited by multiple scattering effects

- Is it feasible to differentiate incident muon momenta based on energy deposited in scintillator?

- Could permit the use of only muons that fall in a "sweet spot" of energies for reconstruction

- Supporting data analysis and visualizations in Root and Paraview 


\section{MC: Detector Design \& Particle Gun}

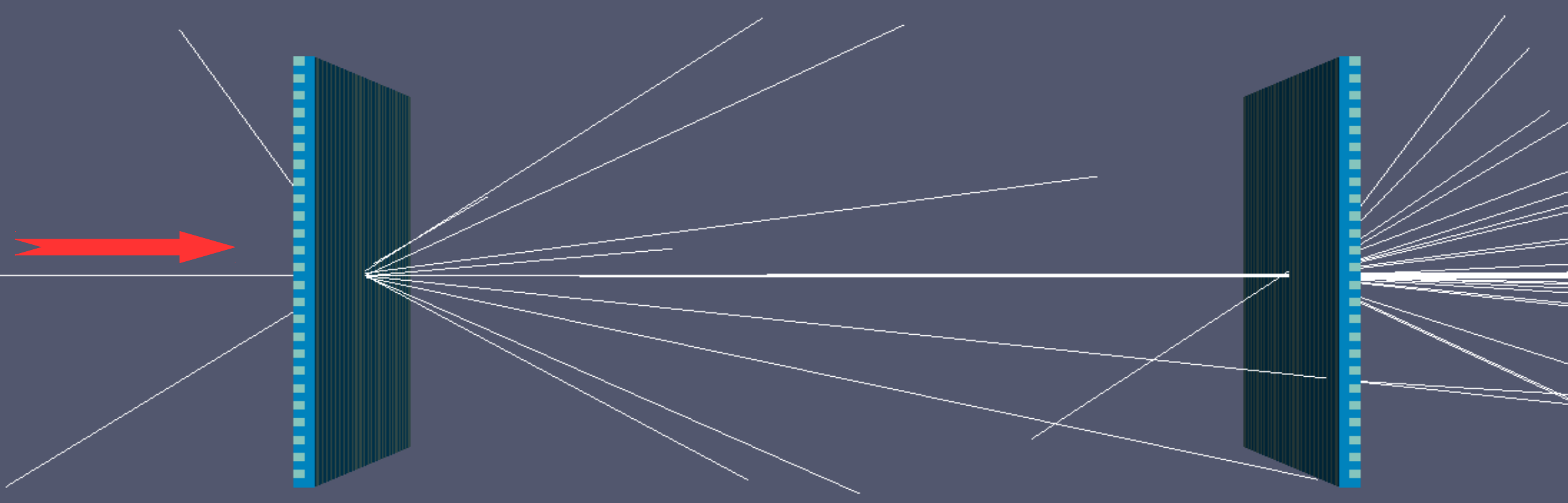

Simulation contains two detectors, each consisting of two $1 \mathrm{~m} \times 1 \mathrm{~m}$ scintillator planes and each plane composed of $502 \mathrm{~cm} \times 2 \mathrm{~cm} \times 1 \mathrm{~m}$ strips; there is a $2 \mathrm{~m}$ gap between the first and second detectors. G4ParticleGun produces 1 muon at a time, with the same initial momentum and position each time. 


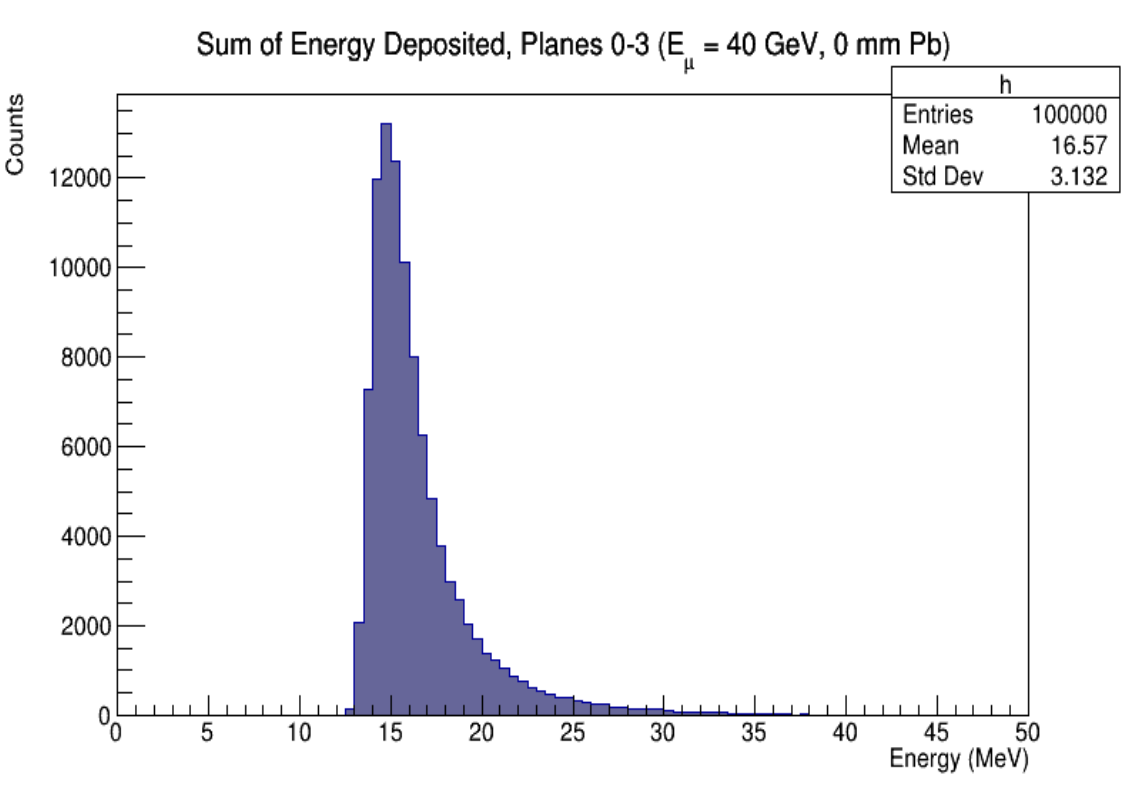

Mean Energy Deposition, Planes 0-3

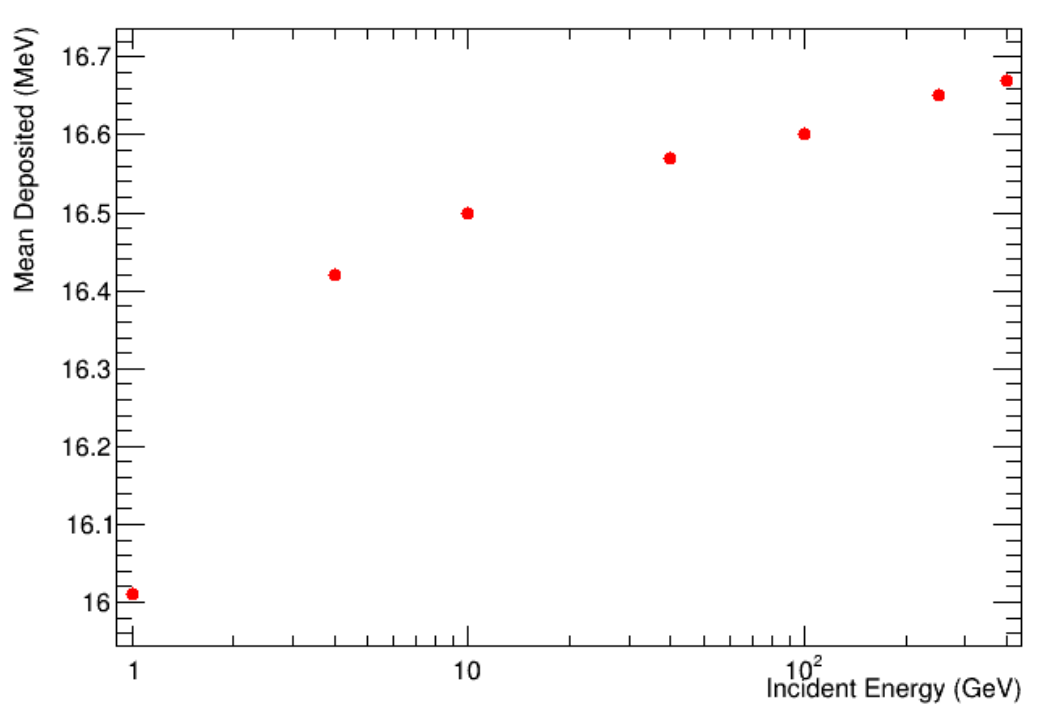

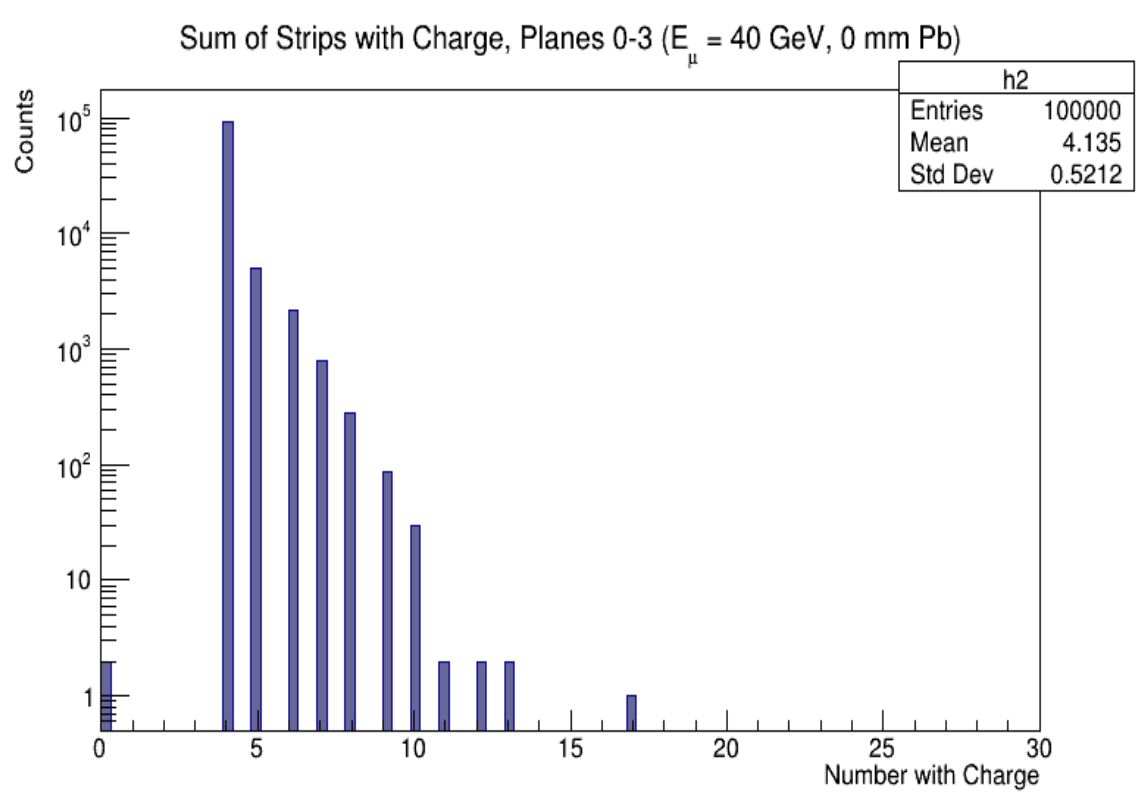

Mean Energy Loss

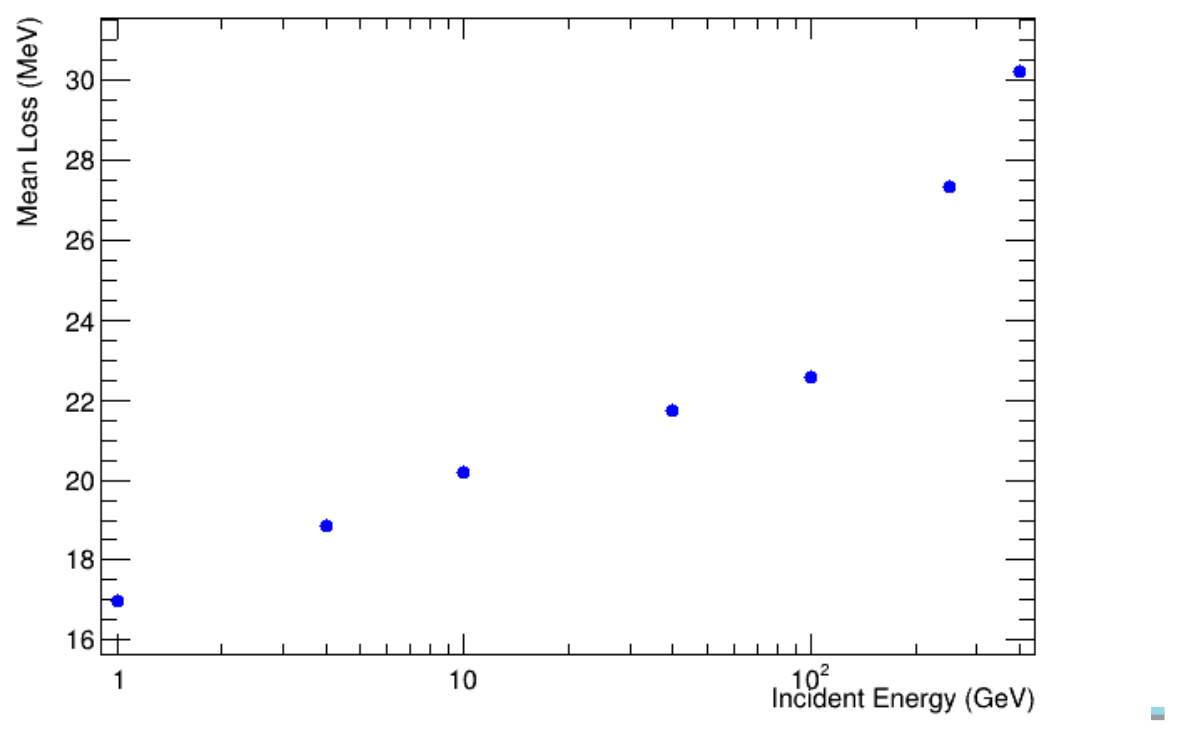




\section{MC: Alternate Detector Design - Pb Layer}

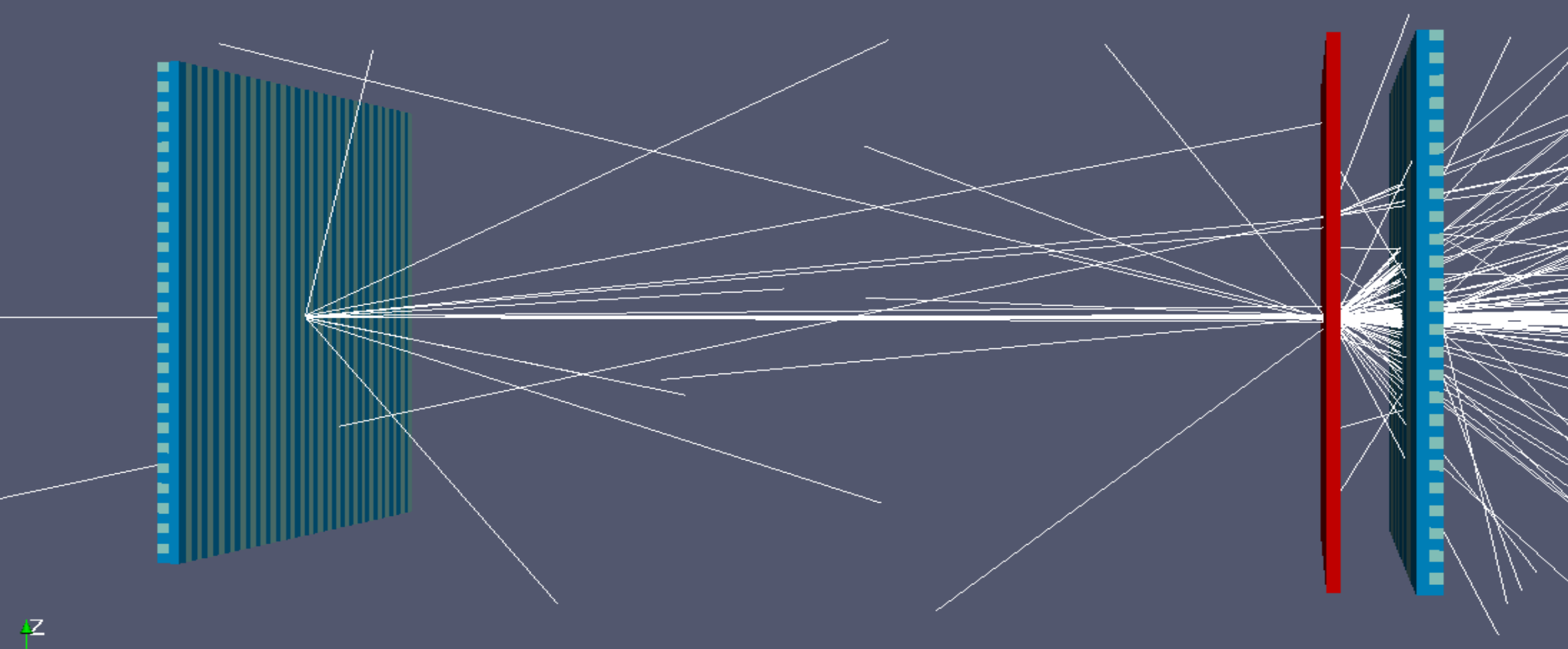

$\Rightarrow x$

The modified detector design includes a lead sheet of varying thickness between the two detector planes, and the placement of this sheet ranged from $12 \mathrm{~cm}-20 \mathrm{~cm}$ upstream of detector 2 . The goal of this addition was to increase the number of secondaries striking detector 2 without degrading position resolution, as this would help differentiate low- and high-energy muons. 


\section{MC: Pb Layer - Energy Results}

Mean Strips Hit in Planes 2-3

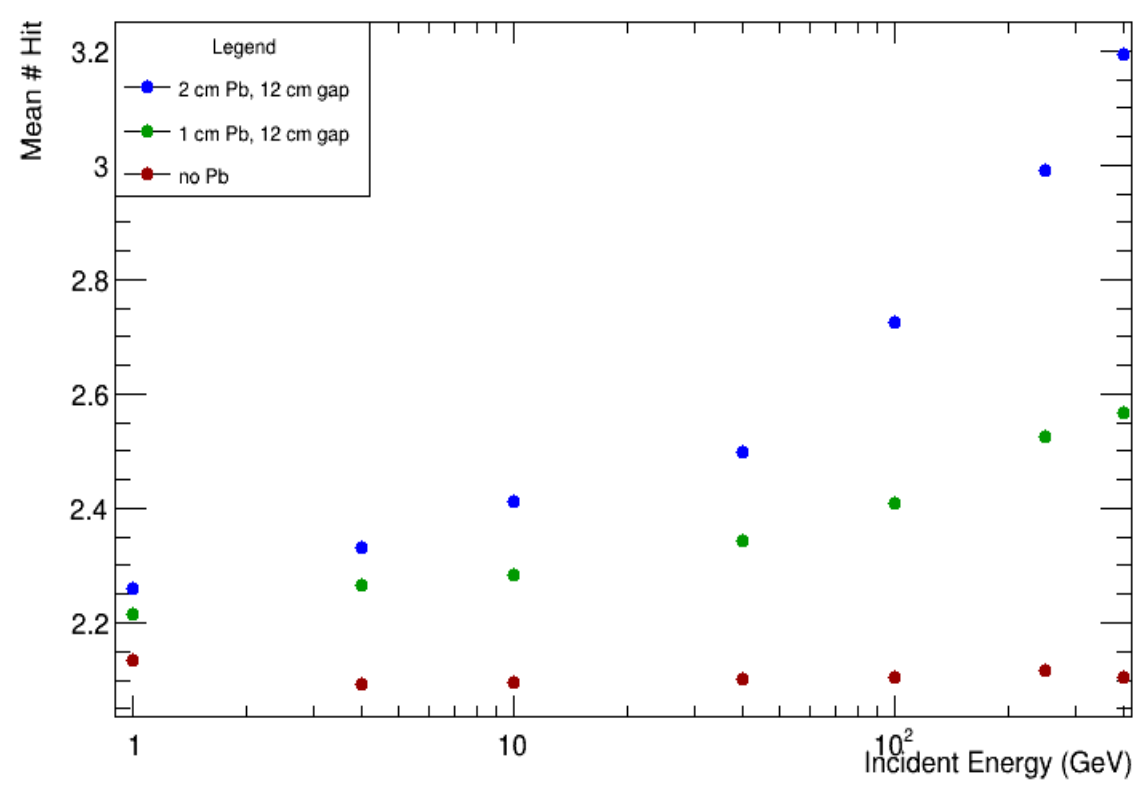

Number of Events with $>10$ Strips Hit in Planes 2-3 (10,000 muons/run)

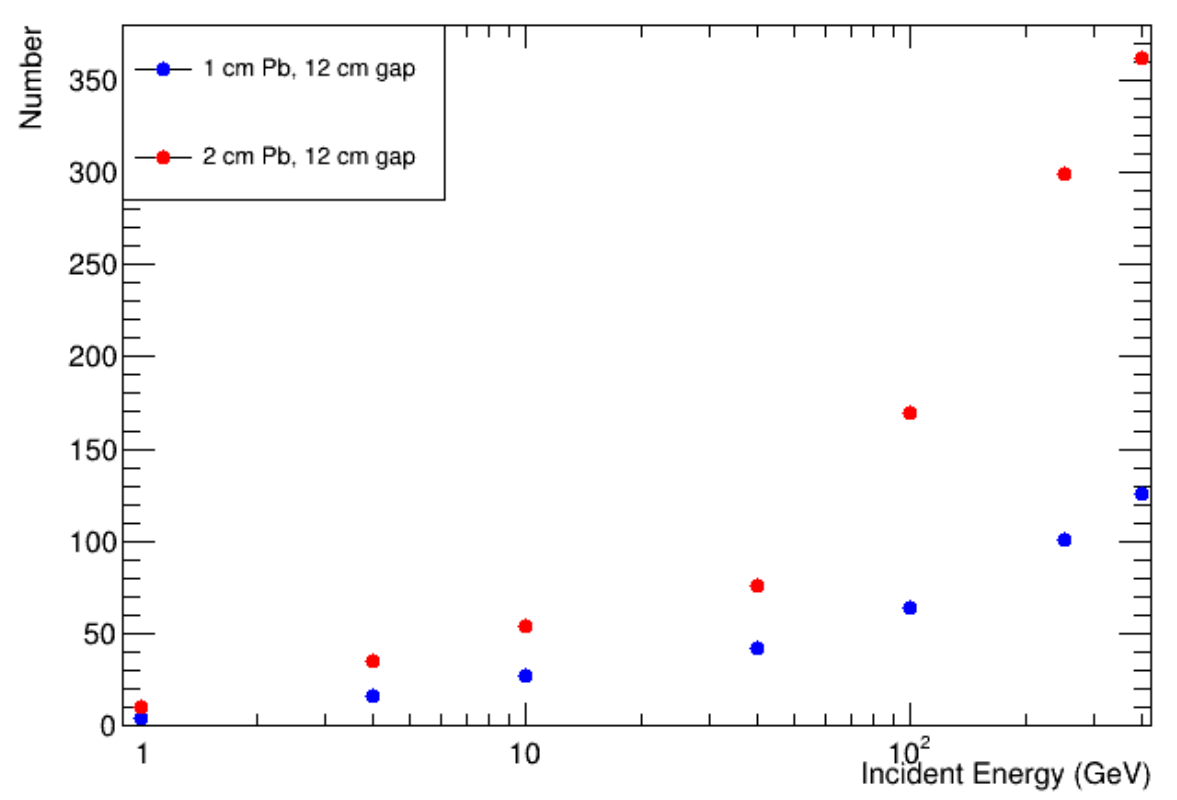




\section{MC: Muon Hit Position \& Angular Resolution}

- The strips hit in each detector provide a point in space which lies on the muon's trajectory

- Can combine hits from both detectors to reconstruct muon's path $\rightarrow$ backproject to predict where muon came from at any desired distance

- RMS error from cell of width $A=A / \sqrt{ } 12$

- What size scintillator strip is needed, given the effects of multiple scattering in the pyramid?

$$
\theta_{0}=\frac{13.6 \mathrm{MeV}}{\beta c p} z \sqrt{x / X_{0}}\left[1+0.038 \ln \left(x / X_{0}\right)\right]
$$




\section{MC: Position Resolution}
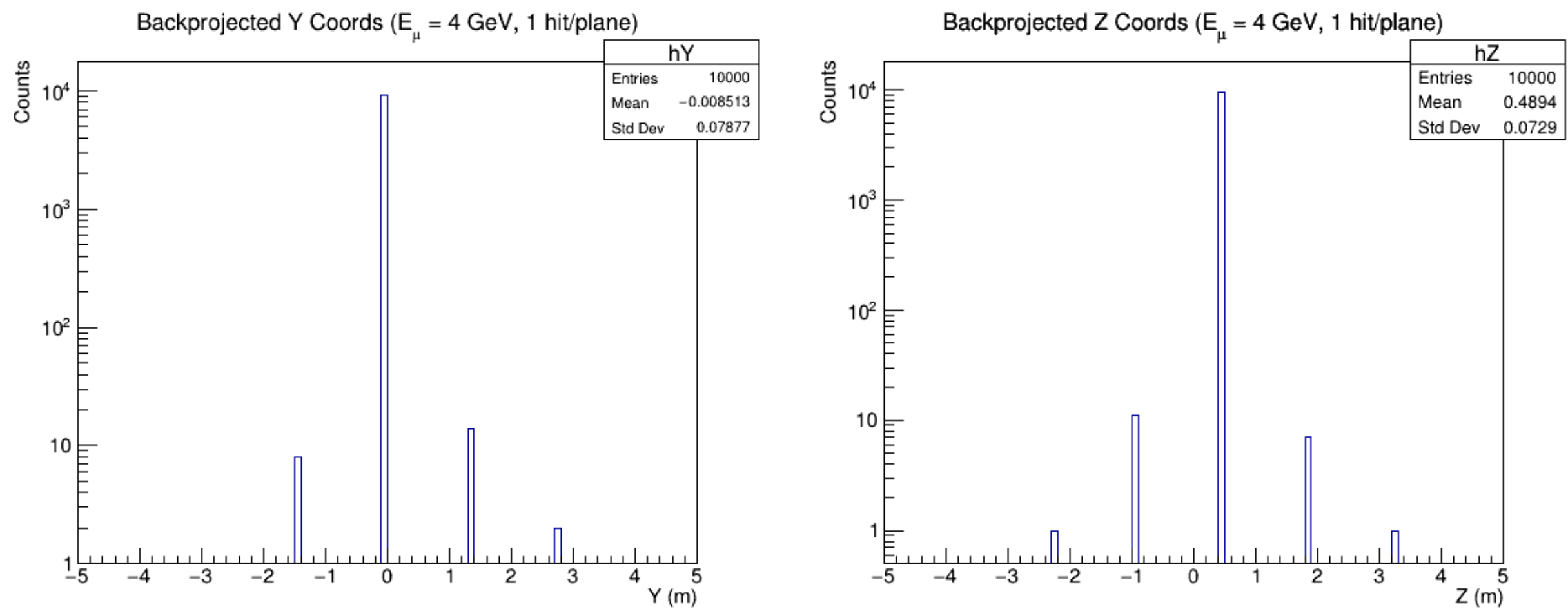

Preliminary results from position resolution algorithm (beam at normal incidence, backprojected a distance of $140 \mathrm{~m}$ ). Non-central bins stem from events in which the muon scattered to a different strip in the detector. 


\section{MC: Future Work}

- Likely not feasible to use number of hits to tag muon energy

- Small increase in mean \# hit over the energy range of interest

- Small fraction of total muons results in this increase

- Continue development of hit position clustering algorithms

- Vary angle of muon beam and repeat angular resolution studies

- Add concrete block as pyramid dummy

- Study effects of multiple scattering on angular resolution

- Optimize scintillator strip size and shape 


\section{Acknowledgments}

My sincere thanks to:

- Alan Bross

- Henry Frisch

- Ralf Ehrlich

- Adam Para

- Anna Pla-Dalmau

- Leah Christine Welty-Rieger

- Omar Shohoud

- The members of the SIST committee

This manuscript has been authored by Fermi Research Alliance, LLC under Contract No. DE-AC02-07CH11359 with the U.S. Department of Energy, Office of Science, Office of High Energy Physics. 


\section{Thank You!}

Image Credits:

https://www.deviantart. com/atomiccheese/art/ Pyramid-SchemeCartoon-200543958

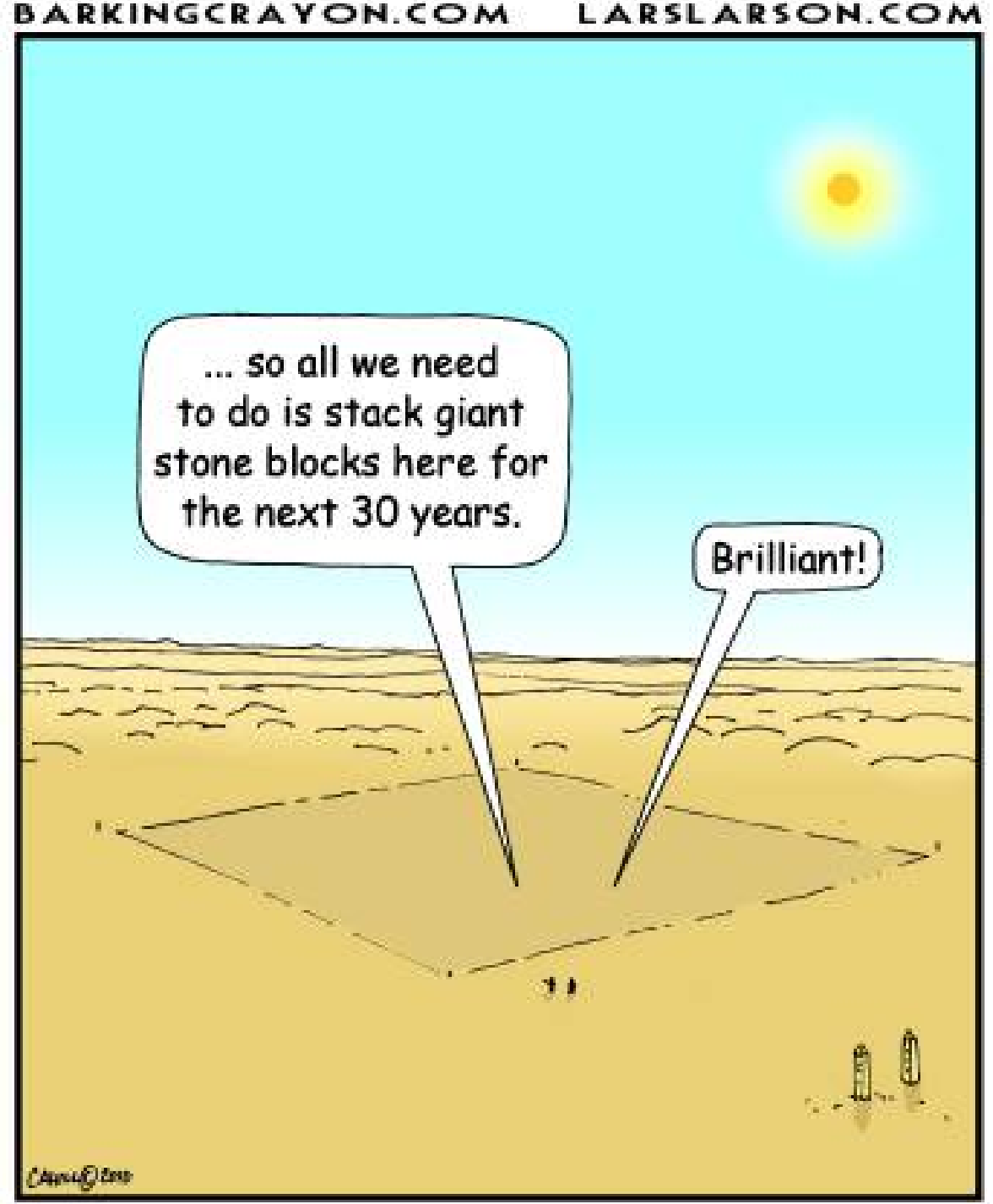

The greatest pyramid scheme of them all 